\title{
Characterization of bioactive substances MHGF-68 on tumour cell lines with LiveFlow In Vitro Technology
}

${ }^{1}$ Comenius University, Faculty of Natural Sciences, Department of Microbiology and Virology, Ilkovičova 6 ,

Mlynská dolina, 842 15, Bratislava 4, Slovak republic

${ }^{2}$ Slovak Academy of Sciences, Center of Biosciences,

Dúbravská cesta 9, 84505 Bratislava, Slovak republic

${ }^{3}$ Comenius University in Bratislava, Faculty of Pharmacy,

Department of Galenic Pharmacy, Odbojárov 10

83232 Bratislava 3, Slovak Republic

Received 11 November, 2020, accepted 22 March, 2021

Abstract As part of experimental research, growth factor-like substances associated with MHV-68, named MHGF-68, were discovered in our laboratory. MHGF-68 activity was manifested by the ability to alter cell morphology, that is, normal phenotype to transformed, resp. suppresses the transformed phenotype of tumour cells. The aim of the experiments was to monitor the effect of MHGF68 on the change of the cell actin cytoskeleton in the tumour cell line Hepa1c1c7, as well as the normal cell line NIH3T3, and compare conventional stationary cultivation and dynamic cultivation conditions using a LiveFlow system (In Vitro Technologies). LiveFlow is an advanced system to test the impact of different compounds on the cell cultures, which allows simulation of in vivo conditions thanks to continuous flow of cultivation medium. MHGF-68 was prepared with the infection of BHK-21 cells with MHV- 68 virus under non-permissive conditions $\left(41^{\circ} \mathrm{C}\right)$. After dynamic cultivation with MHGF- 68 , we observed changes in morphology on Hepa1c1c7 cells. In cells cultured in a dynamic environment, we observed more pronounced changes in cell morphology in comparison with cells cultured statically. We observed no changes in the cytoskeletal structures in the NIH $3 \mathrm{T3}$ cell line affected by MHGF-68 in both types of cultivation. The advantage of LiveFlow in comparison to in vivo testing is that the experiments performed in this system are less time and money consuming. Dynamic cultivation in the LiveFlow system is suitable for optimizing experiments before testing substances in vivo.

Keywords MHV-68-bioactive substances MHGF-68-cytoskeletal structure - LiveFlow system - tumour cell line

\section{INTRODUCTION}

In vitro cultures are a tool for preliminary investigation cell behaviour. They allow to control most of the experimental variables and permit quantitative analysis. In comparison to in vivo, in vitro models are highly controllable, with a reduction in time and cost (Table 1). Common model for in vitro studies is the cell monolayer cultured in static conditions. Ideally, an in vitro model should come as close as possible to the in vivo situation (Giusti et al., 2014). LiveFlow is an advanced system to test the impact of different compounds on the cell cultures, which allows simulation of in vivo conditions thanks to continuous flow of cultivation medium; with the possibility of exact regulation flow speed, IVTech technology is based on compact, user-friendly and transparent cell culture chambers (LiveBox) with shape and dimensions similar to the 24-well plate wells. The main advantage of LiveFlow is the possibility to simulate different tissues and view them in real time, maintaining the same protocols used in traditional cell culture experiments. In this study, we treated cells with MHGF-68 and observed the effect on the cell morphology after cultivation with stationary conditions and after dynamic cultivation resembling in vivo conditions with a LiveFlow system (In Vitro Technologies). MHGF68 compounds are bioactive compounds that resemble cellular growth factors and were isolated in our laboratory in 2015 (Šupolíková et al., 2015). There are already known herpesviruses and poxviruses which have genes encoding secretory proteins with structural similarity to cellular growth factors (Konvalina et al., 2002). The first growth factors related 
Table 1. Comparison of IVTech, in vitro and in vivo testing.

\begin{tabular}{|c|c|c|}
\hline In vitro & In vivo & IVTech (advanced cell culture systems) \\
\hline Lack of human complexity & Ethically controversial & Human organ environment simulation \\
\hline Lack of side effects tests & Time ineffective & Multi-organ models \\
\hline Lack of geometrical complexity & $\begin{array}{c}\text { Expensive }(2-30 \text { times more than in } \\
\text { vitro })\end{array}$ & 3D and dynamic cell cultures \\
\hline Cells cultivated in static conditions & No high-throughput monitoring & Real time monitoring \\
\hline
\end{tabular}

to herpesviruses were obtained from the alphaherpesviruses, specifically pseudorabies virus (PRGF) and herpes simplex virus (HSV) type 1 and type 2 (HSGF-1 and HSGF-2) (Golais et al., 1990; Golais et al., 1992). MHGF-68 compounds were obtained by the cultivation of BHK-21 cells infected with murine herpesvirus-68 (MHV-68) under non-permissive conditions for viral replication (cultivation at $41^{\circ} \mathrm{C}$ ). The viral origin of MHFG-68 was confirmed with a panel of monoclonal antibodies directed against viral glycoprotein B (Šupolíková et al., 2015). These substances are able to change the cell morphology from normal to transformed phenotype and vice versa (Šupolíková et al., 2018). Basic chemical separation of MHGF-68 substances on FPLC column with Sephadex G15 in the absence of salts resulted in loss of transformation activity, while the ability to suppress transformation remained conserved. On the other hand, both effects were conserved when separated on the same column but washed with phosphate buffered saline (PBS). MHGF-68 substances were separated on two biologically active components $\mathrm{MHGF}_{\mathrm{A}}$ and $\mathrm{MHGF}_{\mathrm{B}}$ after separation on the different column (RPHPLC (18) using methanol-water phase. Function of these components is to change the normal cell phenotype to transformed phenotype (Šupolíková et al., 2015).

In our experiments, we have confirmed that MHGF-68 has a two-component character and its $\mathrm{Mr}$ is $<1000$. We used MALDI technique, which is also suitable for checking the samples during the purification procedure for checking the mass of the protein/peptide content after fractionation after RP-HPLC, FPLC respectively (Olejníková et al., unpublished data). HPCL-MS showed that every fraction offers different types and different amount of ions during the ionization, which means that the fractions consist of different types of (bio)chemical substances. Chemometrical techniques showed that there are (bio)chemical substances responsible for biological activity. Detailed structure of those compounds needs further study and subsequent experimental work (Vojs Staňová, personal communication). Based on preliminary, the results of the solid-phase extraction (SPE) experiments show that MHGF-68 biologically active substances contain in its molecule a phenolic structure, as evidenced by this that the substances are ionized only at high $\mathrm{pH}=9$ and $\mathrm{pH}=11$. This fact is also confirmed by the fact that these substances are not captured on the C 8 column, but they are captured on a functional group column propylamine, which behaves as a weak annex. At the same time, these substances, despite ionization, are partially captured on phenyl and cyanide column (Vojs Staňová et al., 2015). The aim of the present study was to compare the effect of MHGF-68 on selected cell lines in conditions of stationary in vitro cultivation and dynamic in vitro cultivation in LiveFlow apparatus.

\section{MATERIALS AND METHODS}

\section{MHGF-68}

MHGF-68 compounds were obtained by the cultivation of BHK-21 cells (baby hamster kidney) infected with murine herpesvirus-68 (MHV-68). Monolayers of BHK-21 cells were cultivated in Dulbecco's modified Eagle's medium (DMEM) enriched with $10 \%$ fetal bovine serum (FBS), $1 \%$ penicillin/ streptomycin, $1 \%$ L-glutamine and $0.1 \%$ gentamicin. Subsequently, cells were infected with MHV-68 with multiplicity of infection (MOI) 0.01 and incubated for 24 hours in $41^{\circ} \mathrm{C}$. Afterwards, cells were transferred to $37^{\circ} \mathrm{C}$ and cultivated for another 24 hours. After cultivations, media from these cells were pooled, lyophilized and stored in $+4^{\circ} \mathrm{C}$ until other experiments were performed (Šupolíková et al., 2015). The fraction of MHGF- 68 used in experiments was acquired by FPLC separation on Sephadex G15 column, eluted by redistilled water, so the transformation suppressing activity remained. Experimental conditions: $5 \mathrm{ml}$ of medium obtained from infected cells MHV-68 was loaded onto an HPLC analyser (Shimadzu, Kyoto, Japan) with column of dimensions $950 \times 20$ mm containing sorbent Sephadex G15 Fine (Pharmacia, Lund, Sweden). Deionized was used as the mobile phase water or saline ( $\mathrm{pH}$ 7.2). Flow mobile phase was $0.8 \mathrm{ml} / \mathrm{min}$. Fractions were collected based on the signal from the UV detector at the wave detectors lengths 220 and 254 nm (Vojs Staňová et al., 2015).

\section{Cells}

Experiments were performed on adherent fibroblast cell lines Hepa1c1c7 and NIH3T3. Cell line Hepa1c1c7 (ATCC ${ }^{\circledR}$ CRL$2026^{\mathrm{TM}}$ ) is a stabilized tumour cell line isolated from murine hepatocellular carcinoma, whereas cell line NIH3T3 is a normal cell line derived from murine embryonal cells (ATCC ${ }^{\circledR}$ CRL1658 $\left.{ }^{\mathrm{TM}}\right)$. Cells were cultivated in DMEM enriched with $7 \%$ 


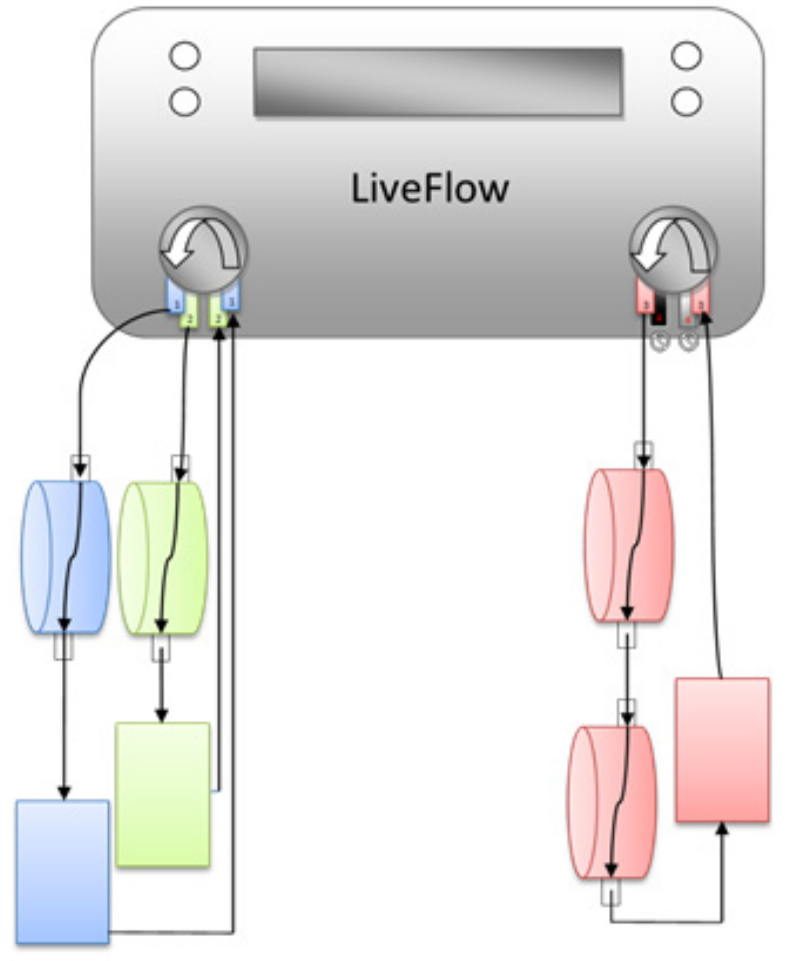

Figure 1. LiveFlow system scheme. Blue and green - LiveBox with samples, parallel circuits of medium, Red - controls, series circuit of medium.

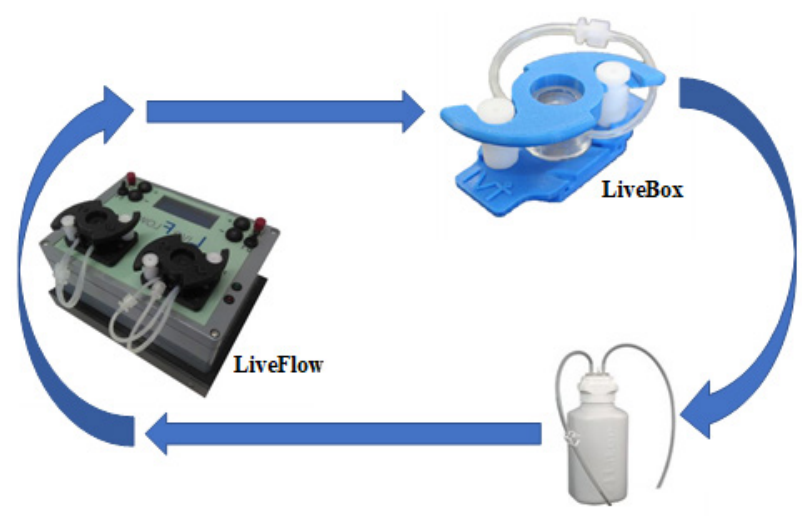

Figure 2. Scheme of dynamic cultivation using LiveFlow (IVTech). LiveBox with adherent cell line on the glass slide inside, reservoir with cultivation medium and LiveFlow ensuring continual flow of the medium.

FBS, 1\% PSA (antibiotics: $100 \mathrm{U} / \mathrm{ml}$ penicillin/streptomycin, $100 \mu \mathrm{g} / \mathrm{ml}$ amphotericin) and $1 \% \mathrm{~L}$-glutamine, and they were incubated in $37^{\circ} \mathrm{C}$ with $5 \% \mathrm{CO}_{2}$.

\section{Cell cultivation in dynamic environment with the use of LiveFlow system}

Sterile circular glass cover slides (20 mm in diameter) were placed in 6-well plates ( 1 slide per well). 1 × $10^{5}$ cells were seeded onto glass slides in volume of $0.25 \mathrm{ml}$ per slide. After a 20-minute-long incubation, $2 \mathrm{ml}$ of DMEM was added to each well. After a 24-hour-long cultivation, glass slides were placed into LiveFlow system, as shown in Figure 1, and the cultivation medium with MHGF-68 (diluted 1:100) was added. In every experiment, control untreated cells (without MHGF68) were used. Volume of circulating medium was $7.5 \mathrm{ml}$ per LiveBox ( 1 slide). Cells were cultivated 24, 48 and 72 hours with continuous flow of medium at a speed of $180 \mu \mathrm{l}$ per minute (Figure 2). Incubation was performed at $37^{\circ} \mathrm{C}, 5 \% \mathrm{CO}_{2}$. For information: LiveFlow system is commercially manufactured by In Vitro Technologies (in short IVTech) and distributed by Scintilla spol. s r.o.

\section{Cell cultivation in static conditions}

Sterile circular glass cover slides (20 $\mathrm{mm}$ in diameter) were placed in 6-well plates ( 1 slide per well). $1 \times 10^{5}$ cells were seeded onto glass slides in volume of $0.25 \mathrm{ml}$ per slide. After a 20-minute-long incubation, $2 \mathrm{ml}$ of DMEM was added to each well. After a 24-hour-long cultivation, the medium was exchanged with a medium with MHGF-68 (diluted 1:100). In every experiment, control untreated cells (without MHGF-68) were used. Cells were cultivated for 24,48 and 72 hours at $37^{\circ} \mathrm{C}, 5 \% \mathrm{CO}_{2}$.

\section{Immunofluorescence assay}

After incubation, the cells were fixed with $4 \%$ paraformaldehyde solution and permeabilized with $0.01 \%$ Triton X-100 (PBS solution). Alexa Fluor 555 conjugated with Phalloidin was used to visualize actin filaments. To visualize cell nuclei, DAPI stain was used. Prepared slides were observed using Leica TCS SP8 AOBS (Leica Microsystems, Germany) confocal microscope with HC PL APO CS2 63x/1.40 OIL lens.

\section{RESULTS AND DISCUSSION}

The cytoskeleton plays an important role in the regulation of various cellular processes associated with transformation, such as proliferation, contact inhibition, and cell growth in thin agar or apoptosis. In general, loss of actin filaments is considered a marker of oncogenicity (Pawlak and Helfman, 2001; Etienne-Manneville, 2004; Shutova and Alexandrova, 2010). In this study, morphological changes in cellular actin cytoskeleton after treatment with bioactive substances MHGF-68 were observed. These changes were significantly more pronounced on Hepa1c1c7 cells, especially after dynamic cultivation in LiveFlow system. After dynamic cultivation in LiveFlow, the shape of untreated (control) cells was rhomboidal (Figure 3A, B, C). Actin cytoskeleton of untreated cells was diffusive, probably depolymerized, with small aggregations of actin molecules with filamentous actin near cell margin (Figure 3C). Cells treated with MHGF-68 after 24 hours of cultivation had enlarged nuclei and changed 

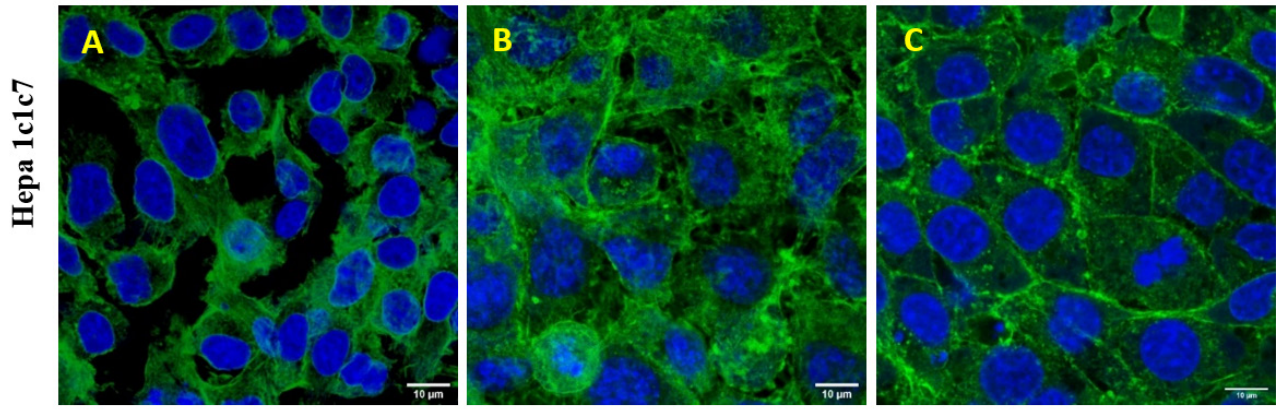

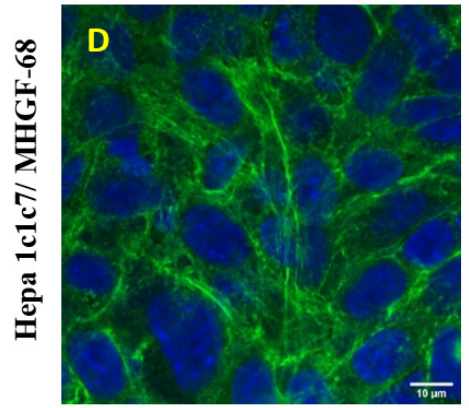

$\mathbf{0 ~ h}$

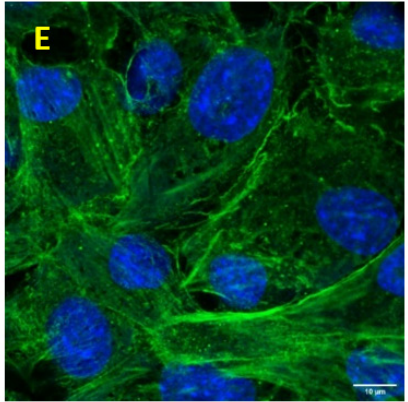

$24 \mathrm{~h}$

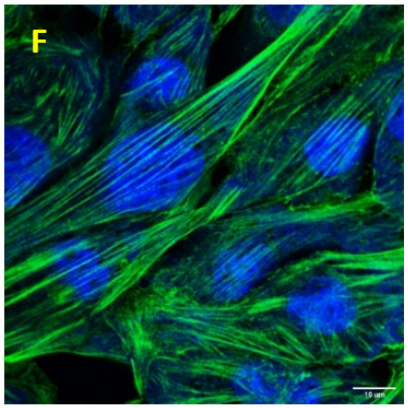

$72 \mathrm{~h}$

Figure 3. Distribution of actin filaments in Hepa1c1c7 cells incubated with MHGF-68 after dynamic cultivation.

Hepa 1 c 1 c 7 cells were incubated with MHGF-68. After 0, 24 and $72 \mathrm{~h}$, the cells were fixed and labeled with Alexa Fluor 555 phalloidin. Nuclei were stained with DAPI. The intracelular distribution of actin filamets (green) and nuclei (blue) were imaged by confocal laser scanning fluorescence microscopy (Leica TCS SP8 AOBS) with HC PL APO CS2 63x/1.40 OIL lens.

A Hepa1c1c7 untreated, 0 h cultivation B Hepa1c1c7 untreated, 24 h cultivation C Hepa1c1c7 untreated, 72 h cultivation D Hepa1c1c7 + MHGF-68, 0 h cultivation E Hepa1c1c7 + MHGF-68, 24 h cultivation F Hepa1c1c7 + MHGF-68, 72 h cultivation

shape (Figure 3E). After 72 hours, cells gained spindle-like shape (Figure 3F). On the contrary, highly organized actin filaments and high granulation of cytoplasm were found in whole-cell volume of cells incubated with MHGF-68 (Figure 3D). Any significant changes in NIH 3T3 cells were not detected. In comparison with dynamic cultivation, cytoskeleton structure of Hepa1c1c7 cells after static cultivation was different. Highly organized actin filaments were not present in the whole volume of cells, but were largely arranged along the edges of the cells (Figure 4E, F). After 72 hours of static cultivation, signs of spindle-shaped cell formation were observed (Figure 4F). On the normal NIH 3 T3 cell line cultured with MHGF-68, we did not observe any changes in the cell cytoskeleton during the dynamic culture (data not shown). Comparison of 72 hours static and dynamic cultivation of Hepa1c1c7 with MHGF-68 showed that during stationary cultivation, changes were less visible (Figure 5). In the case of untreated cells, the formation of highly organized actin filaments and the enlargement of nuclei were observed only after dynamic cultivation in LiveFlow (Figure 5D).

Our results from monitoring the effect of MHGF-68 on cell proliferation, metabolic activity and viability of the cells were confirmed by xCELLigence analyser too (Šupolíková et al., unpublished data). We found that the effect of the MHGF-68 fractions was relatively short-lived and decreased with longer time of cultivation. Since the chemical structure of MHGF-68 fractions is not yet known, the described biological activities require further studies.

\section{CONCLUSION}

Changes of cell size and shape and distribution of actin filaments were observed during both types of cultivation in normal and tumour cell line. Comparison of stationary and dynamic cultivation showed that the effect of MHGF-68 on cytoskeletal structures is more significant during dynamic cultivation. Regarding quantitative determination of the activity of bioactive compounds MHGF-68, it is more suitable to use stationary cultivation. We confirmed by MTT assay that the effect of MHGF-68 in a static cultivation showed a significant inhibitory effect on the proliferation and viability of NIH 3T3 and Hepa1c1c7 cells and that the effect in the dynamic cultivation did not have a significant effect on the proliferation and viability of Hepa1c1c7 cells (data not shown). However, dynamic cultivation in LiveFlow system is suitable for the optimization of experiments before in vivo tests on laboratory animals, as it simulates conditions in live organisms by continuous flow of culture medium. 

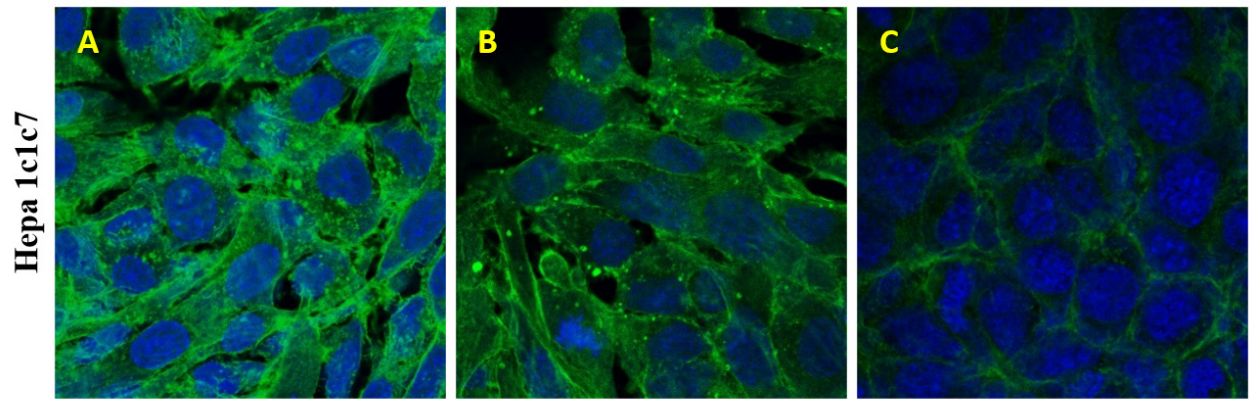

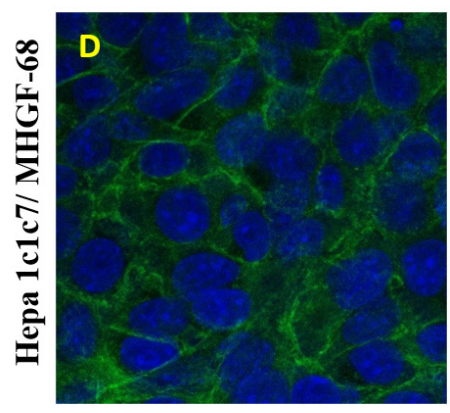

$\mathbf{0 ~ h}$

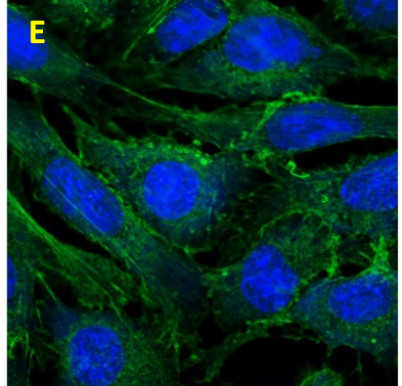

$24 \mathrm{~h}$

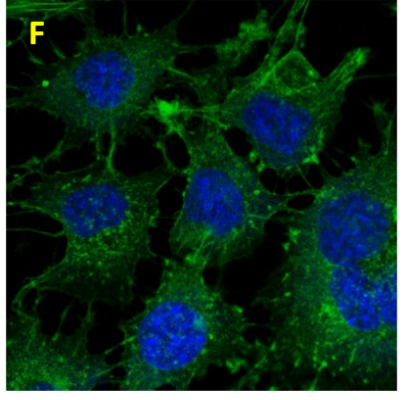

$72 \mathrm{~h}$

Figure 4: Distribution of actin filaments in Hepa1c1c7 cells incubated with MHGF-68 after static cultivation.

Hepa1c1c7 cells were incubated with MHGF-68. After 0, 24 and 72 h, the cells were fixed and labeled with Alexa Fluor 555 phalloidin. Nuclei were stained with DAPI. The intracelular distribution of actin filamets (green) and nuclei (blue) were imaged by confocal laser scanning fluorescence microscopy (Leica TCS SP8 AOBS) with HC PL APO CS2 63x/1.40 OIL lens. A Hepa1c1c7 untreated, 0 h cultivation B Hepa1c1c7 untreated, 24 h cultivation C Hepa1c1c7 untreated, 72 h cultivation D Hepa1c1c7 + MHGF-68, 0 h cultivation E Hepa1c1c7 + MHGF-68, 24 h cultivation F Hepa1c1c7 + MHGF-68, 72 h cultivation
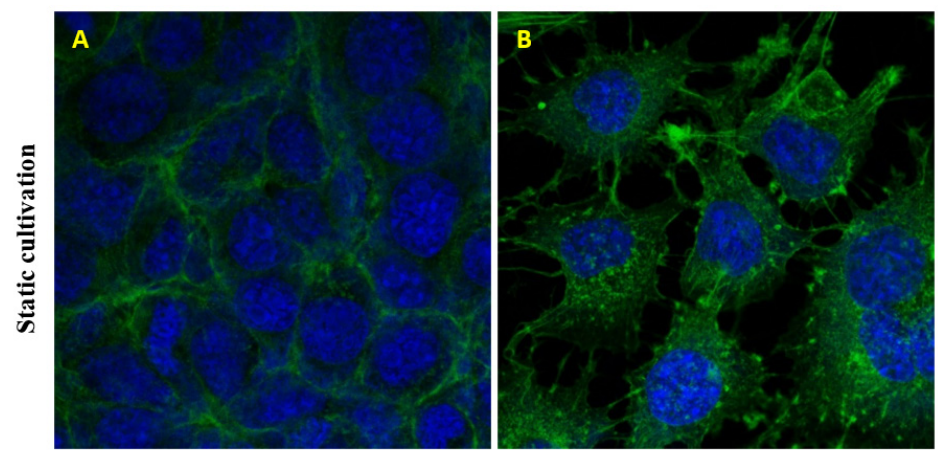

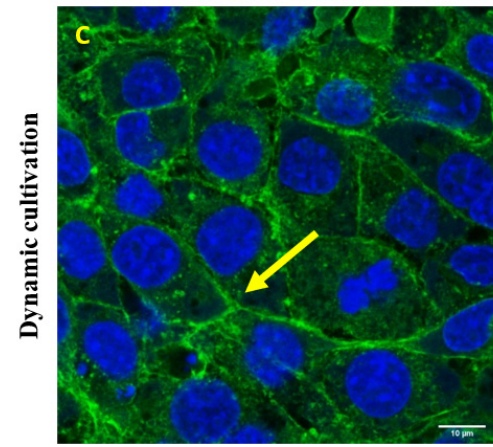

Hepa 1c1c7

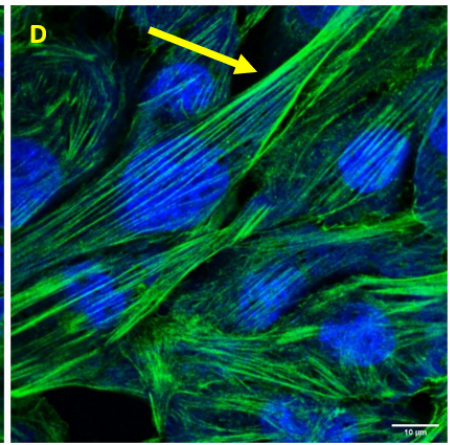

Hepa 1c1c7/ MHGF-68

Figure 5. Distribution of actin filaments in Hepa1c1c7 cells incubated $72 \mathrm{~h}$ with MHGF-68 after static and dynamic cultivation. Hepa1c1c7 cells were incubated with MHGF-68. After $72 \mathrm{~h}$, the cells were fixed and labeled with Alexa Fluor 555 phalloidin. Nuclei were stained with DAPI. The intracelular distribution of actin filamets (green) and nuclei (blue) were imaged by confocal laser scanning fluorescence microscopy (Leica TCS SP8 AOBS) with HC PL APO CS2 63x/1.40 OIL lens.

A Hepa1c1c7 untreated, static cultivation B Hepa1c1c7 + MHGF-68, static cultivation C Hepa1c1c7 untreated, dynamic cultivation D Hepa1c1c7 + MHGF-68, dynamic cultivation 


\section{ACKNOWLEDGEMENTS}

This research was carried out with financial support from VEGA 1/0061/18, APVV-0621-12 and OPVaV-2015/3.1/01SORO.

\section{References}

[1] Etienne-Manneville S. Actin and microtubules in cell motility: which one is control? Traffic. 2004; 5: 470-477.

[2] Giusti S, Sbrana T, La Marca M, Di Patria V, Martinucci V, Tirella A, Domenici C, Ahluwalia A. A novel dual-flow bioreactor simulates increased fluorescein permeability in epithelial tissue barriers. Biotechnol J. 2014; 9: 1175-1184.

[3] Golais F, Koštál M, Csabayová M, Leško J. The glycoprotein B gene and its syn 3 locus of herpes simplex virus type 1 are involved in the synthesis of virus-associated growth factor (HSGF-1). Acta Virol. 1992; 36: 505-515.

[4] Golais F, Leško J, Hillerova A, Sabo A, Kolcunova A. A putative virus-encoded growth factor in a crude extract of pseudorabies virus infected and transformed cells. Biologisches Zentralblatt 1990; 109: 481-487.

[5] Konvalina I, Gašperík J, Golais F. A novel class of growth factors related to herpesviruses. Acta Veterinaria Brno 2002; 71: 29-36.

[6] Pawlak G, Helfman DM. Cytoskeletal changes in cell transformation and tumorigenesis. Curr Opin Genet Dev.2001; 11: 41-47.

[7] Shutova MS, Alexandrova AY. Normal and transformed fibroblast spreading: role of microfilament polymerization and actinmyosin contractility. Cell Tissue Biol.2010; 4: 25-35.

[8] Šupolíková M, Labudová M, Nováková E, Vojs Staňová A, Šišovský V, Golais F. The effect of Murine Herpesvirus 68 (MHV-68) Related Growth Factor 68 (MHGF-68) on the Tumor progression in Athymic Nude Mice Am. J. Biomed. Life Sci. 2018; 6(6): 127-131.

[9] Šupolíková M, Vojs Staňová A, Kúdelová M, Marák J, Zelník V, Golais F. Cells transformed by murine herpesvirus 68 (MHV68) release compounds with transforming and transformed phenotype suppressing activity resembling growth factors. Acta Virol. 2015; 59(4): 418-422.

[10] Vojs Staňová A, Šupolíková M, Koiš P, Golais F, Marák J. Analýza a identifikácia biologicky aktívnych látok indukovaných myším herpetickým vírusom (Analysis and identification of biologically active substances induced by murine herpes virus). Zdravotnícke listy $2015 ; 3(4): 110-114$. 